\title{
Effect of Health Education on Mothers' Adherence to Childhood Immunisation Schedule at Dunkwa-On-Offin in the Upper Denkyira East Municipality of Ghana
}

\author{
Sylvia Ahinee Adjedu \\ Community Health Nurses Training College-Tanoso, Post Office Box 2066, Tanoso, Ghana
}

\begin{abstract}
Immunisation is one of the most essential and cost-effective public health interventions for the prevention of childhood sicknesses and disabilities and a basic need for all children. The aim of this research study was to assess the impact of health education on mother adherence to childhood immunisation schedule a study at Dunkwa-On-Offin in the Upper Denkyira East Municipality of Ghana. This research used was a cross-sectional study and engaged purposive and simple random sampling techniques. The sample size for the study was 70 consisting of mothers and caregivers with children under 24 months and structured interview were the research instrument. From the study findings, all the respondents $70(100 \%)$ stated yes health education provided by health workers has influenced them to adhere to childhood immunisation schedule and $66(94.2 \%)$ respondents said yes they had completed or still running children's immunisation schedule and $2(2.9 \%)$ respondents said to they could not achieve it. The study concluded that health education on mother adherence to childhood immunisation schedule has positively impacted on high mother adherence to childhood immunisation schedule and recommended that Reproductive and Child Health/Family Planning staff should embark on routine education campaigns.
\end{abstract}

Keywords: Immunisation, health education, public health, vaccine coverage

DOI: $10.7176 / \mathrm{JMPB} / 70-01$

Publication date: April $30^{\text {th }} 2021$

\section{Background}

Immunisation is one of the most essential and cost-effective public health interventions for the prevention of childhood sicknesses and disabilities. It is noted that children are the most vulnerable among age groups in the range of disease prevention and have higher morbidity and mortality rate. Vaccine-preventable diseases comprise of but are not limited to measles, polio, whooping cough, tetanus, diphtheria, meningitis, yellow fever, hepatitis B, tuberculosis, pneumonia, diarrhoea and cervical cancers. Among these diseases, measles remains one of the primary causes of death among young children worldwide, notwithstanding the availability of a safe and effective vaccine (World Health Organisation, 2011).

WHO (2019) stresses the role played by immunisation as one of the interventions with a huge potential impact on health outcomes and seen as one of the most effective means of reducing under-five (U-5) mortality rates. For instance, in 2018 it is estimated that in 6.2 million children and adolescents below the age of 15 years died, typically from preventable causes. Of these deaths, 5.3 million happened in the first 5 years, with almost half of these in the first month of life. Primary causes of death in children under-5 years are preterm birth complications, pneumonia, birth asphyxia, congenital anomalies, diarrhoea and malaria. Nearly half of these deaths are in newborns (WHO, 2019).

WHO (2019) further maintains that more than half of these early child deaths are preventable or can be treated with simple, inexpensive interventions including immunisation, adequate nutrition, safe water and food and proper care by a trained health provider when needed. Children in sub-Saharan Africa are more than 15 times more likely to die before the age of 5 than children in high income countries (WHO, 2019). It is argued that Africa has made incredible improvements immunising children against preventable diseases such as polio, pneumonia, diphtheria, measles and tuberculosis. As of $2014,77 \%$ of the continent's children were immunised. Compared to 1980 , when only $5 \%$ of African children were vaccinated, this is a strong progress (Wiysonge, 2015).

Notwithstanding these achievements, the immunisation agenda in Africa remains incomplete (Machingaidze, Wiysonge and Hussey, 2013). Huge numbers of children are still not vaccinated and die from vaccine-preventable diseases. Additionally, WHO (2019) projected that approximately three million children under five years of age die every year in the African Region and a substantial number of these deaths could be prevented by vaccines. Yet one in five children does not receive them. It is suggested inadequate service delivery in distant and inaccessible areas or lack of information about the effectiveness of vaccines is a major reason many do not receive them.

In 1974, WHO introduced the Expanded Programme on Immunization (EPI) to make vaccines accessible to all children globally (WHO, 1974). Ghana introduce its EPI in 1978 and became active countrywide in 1985 
with the objective of increasing immunization coverage from 6 to $80 \%$ among children $<1$ year of age against six target diseases: Diphtheria, Tetanus, Pertussis, Poliomyelitis, Tuberculosis, and Measles (2002) Ghana Health Service. These diseases have been added to the first six: Yellow fever, Haemophilus influenza type B, Hepatitis B, Rotavirus diarrhoea and Pneumonia (Ghana Health Service, 2012). Aftermath of the EPI execution in Ghana, children $<5$ years of age mortality has dropped increasingly but still needs more effort and attention. According to 2014 Ghana Demographic and Health Survey (GHDS) report, infant mortality rates have declined marginally from 64, 50 and 41 deaths per 1000 live births while under five mortality rates also dropped from 111, 80 and 60 per 1000 live births over a couple of years 2003, 2008 and 2014 correspondingly (GHDS, 2014).

There should be a continuous increase in quality of the EPI programme and reduction in under-five morbidity and mortality. In Ghana some of the measures that have been put in place to improve the effectiveness of immunisation in areas of vaccine supply and quality, in service training and capacity building, improving logistics and resources, surveillance and communication. Health education is one strategy that Ghana Health Service Health is using to promote complete child immunisation. While some research has been carried out on possible indicators for low immunisation, there have been few empirical investigations into influence of health education on mother adherence to childhood immunisation. This study, therefore assess the effect of health education on mother adherence to childhood immunisation schedule in selected towns in the Upper Denkyira East Municipality.

\section{Methods}

A cross-sectional study was used assess the impact of health education on mother adherence to childhood immunisation schedule. The cross-sectional survey design was employed since the researchers were interested in the views of mother or caregivers and health workers providing comprehensive immunisation schedule services. Cross-sectional research study is grounded on observations that take place in different groups at one time. This means there is no experimental method, so no variables are manipulated by the researchers.

The study population included all mothers or caregivers with children under 24 months within the catchments area of Dunkwa-On-Offin who consented to participate in the study. The inclusion criterion was participants residing catchment area and consenting to be part of the study. The exclusion criterion was location outside Dunkwa-On-Offin. This study engaged purposive and systematic sampling techniques. Purposive sampling was used to 70 mothers and caregivers with children under 24 months. Simple random sampling was used to select the participants.

Structured interview was the main data collection technique used in this study. The researcher administered the structured interview for all the participants with children under 24 months. The structured interview offered a richer, more comprehensive view of the study objective and easily repeated to check the reliability of the data.

\section{Ethical Consideration}

Ethical clearance was sought from the Municipal Director of Health Services for Upper Denkyira East Municipality of Ghana. The consent of the participants was subsequently sought. This was preceded by briefing the participants regarding their role and conducts and expectations of the research team. Confidentiality was assured since they were informed not to provide their names or contact to the researchers. Researcher also gave the participants the opportunity to seek clarifications for any uncertainties that they had.

\section{Results}

Demographic Characteristics of mothers/caregivers

Seventy mothers/caregivers were selected for the study and from Table 1 below, respondents within the ages of 21-30 years were $33(47.1 \%)$ of the respondents, those within the ages of 31-40 years were $20(28.6 \%)$, those below 20 years were $10(14.3 \%)$ whereas those within the ages of $41-50$ years were $7(10 \%)$. Concerning the marital Status of the mothers/caregivers, $50(71.4 \%)$ respondents were married, $14(20 \%)$ were single and 6 $(8.6 \%)$ were divorced. In terms of respondents religious attachment, $61(87.1 \%)$ of the respondent were Christians, $7(10 \%)$ were Moslems while $2(2.9 \%)$ were traditionalist. With regards to the respondents' educational background, $41(58.6 \%)$ of the respondents had basic education, $18(25.7 \%)$ respondents had secondary education and those with tertiary education and no formal education were $7(10 \%)$ and $4(5.7 \%)$ respectively. 
Knowledge Level of Mothers on Adherence to Childhood Immunisation Schedule

Table 4.1: Mothers/caregivers' knowledge childhood immunisation schedule

\begin{tabular}{lcc}
\hline Variable & $\mathrm{N}=70$ & \\
\hline Age for schedule completion & 57 & 81.4 \\
One year & 8 & 11.4 \\
One year six months & 4 & 5.7 \\
Two years & 1 & 1.4 \\
More than two years & & \\
Age for Dosage booster & 58 & 82.9 \\
One-five years & 10 & 14.3 \\
One and half-five years & 2 & 2.7 \\
Two- five years & & \\
Importance of Immunisation & 70 & 100 \\
Yes & 0 & 0 \\
$\quad$ No & 70 & 100 \\
Diseases immunisation Prevent & 0 & 0 \\
Yes & & \\
No & 70 & 100 \\
Social Mobilisation & 0 & 0 \\
Yes & & \\
No & 60 & 85.7 \\
Satisfaction of immunisation services & 10 & 14.3 \\
$\quad$ Yes & & \\
No & & \\
\hline Souc: Fild & & \\
\hline
\end{tabular}

Source: Field Survey, 2019

Respondents' knowledge on the age at which immunisation schedule is to be completed for child against childhood killer diseases was sought. According to the study results, $57(81.4 \%)$ respondents indicated one year duration, $8(11.4 \%)$ thought one year six months, $4(5.5 \%)$ respondents stated two years and $1(1.4 \%)$ respondents specified more than two years. Concerning the child's age to receive booster dosage against diseases, 58 respondents forming $82.9 \%$ said immediately after one year, 10 respondents forming $14.3 \%$ said immediately after one and half years and 2 respondents forming $2.7 \%$ said immediately after two years.

All the respondents $(100 \%)$ said yes, immunisation is very important and again all the respondents indicated yes meaning that immunization prevent childhood killer diseases. All the respondents $(100 \%)$ said there was social mobilisation for immunisation schedule in their locality. Regarding immunisation service satisfaction, 60 $(85.7 \%)$ respondents were satisfied with the services rendered to them and $10(14.3 \%)$ were not satisfied.

Sources of Health Education for Mother Adherence to Childhood Immunisation

Table 2: Sources of health education for mother/caregiver

\begin{tabular}{lcc}
\hline Variable & $\mathrm{N}=70$ & \\
Seen Vaccination messages & Frequency & Percentage \\
Yes & 70 & 100 \\
No & 0 & 0 \\
Main sources of education & 48 & 68.6. \\
Health facility & 2 & 2.9 \\
Town announcers & 10 & 14.3 \\
Radio/FM & 8 & 11.4 \\
Family members & 2 & 2.9 \\
Friends & & 100 \\
Use of Understandable Language & 70 & 0 \\
Yes & 0 & \\
No & & 90 \\
Place of immunisation session & 63 & 10 \\
RCH & 7 & \\
CHPS & & \\
\hline Sourc: Fim & & \\
\hline
\end{tabular}

Source: Field Survey, 2019

Respondents were asked if they have heard or seen messages about vaccination in the last month and all the $70(100 \%)$ respondents stated yes. On the subject of the main source of information on immunization schedule of 
respondents' children, 48 (68.6\%) obviously said health facility, $10(14.3 \%)$ respondents indicated that radio was their sources of uinformation, $8(11.4 \%)$ respondents indicated family members, $2(2.29 \%)$ respondents each said town announcers and friends respectively. All the $70(100 \%)$ respondents held that they understood the Twi (Akan) language used to educate them on importance of immunisation. In terms of the place respondents went for immunization sessions, $63(90 \%)$ of the respondents mentioned Reproductive Child Health facility and 7 (10\%) respondents mentioned CHPS.

Challenges Affecting the Implementation of Health Education Programme on Adherence to Childhood Immunisation Schedule

Table 3: Challenges facing Mothers/caregivers

\begin{tabular}{lcc}
\hline Variable & $\mathrm{N}=70$ & \\
Fime to immunisation Centre & 52 & Percentage \\
\hline Less than 1hour & 16 & 74.3 \\
1 hour-2hours & 2 & 22.9 \\
More than 2hours & & 2.9 \\
Means to immunization Centre & 66 & 94.2 \\
Foot & 2 & 2.9 \\
Vehicle & 2 & 2.9 \\
Others & & \\
Reason for Incomplete Vaccination & 17 & 24.3 \\
Family activities & 31 & 44.3 \\
Poor health & 8 & 11.4 \\
Forgetting date & 14 & 20 \\
Social engagement & & \\
Adequate Staff & 30 & 42.9 \\
Yes & 40 & 57.1 \\
No & & \\
\hline Source: Field Survey
\end{tabular}

Source: Field Survey, 2019

Respondents indicated the time they spent to reach an immunisation centre for services. According to Table 3, $52(74.3 \%)$ respondents indicated they spent less than 1 hour, $16(22.9 \%)$ indicated they spent 1 hour to 2 hours and $2(2.9 \%)$ indicated that they consumed more than 2 hours. Respondents' means of getting to the nearest immunization centre, $66(94.2 \%)$ respondents said they attended child welfare clinic by foot because, scheduled clinics are closed to them. Two (2.7\%) respondents each said they used vehicle and other means respectively to attend child welfare clinic. In terms of the reason for incomplete vaccination among nursing mothers or carers in the respondents' community, $31(44.3 \%)$ respondents attributed it to poor health during the appointment dates assigned to them, $17(24.3 \%)$ respondents attributed it to being engaged in tight family activities, $14(20 \%)$ respondents also attributed it to various social engagement whereas $8(11.4 \%)$ respondents linked it to forgetting date of immunisation schedule. In relation to the question on adequate staff providing immunisation schedule services, $40(57.1 \%)$ indicated that health staff are not adequate implying more staff were needed and $30(42.9 \%)$ stated that health staff are adequate.

Impact of Health Education on Mother Adherence to Childhood Immunisation Schedule

Table 4: Mothers/caregivers' view on health education

\begin{tabular}{lcc}
\hline Variable & $\begin{array}{l}\mathrm{N}=70 \\
\text { Frequency }\end{array}$ & Percentage \\
\hline Influence of health education & 70 & 100 \\
Yes & 0 & 100 \\
No & & \\
Completion of immunization & 66 & 94.2. \\
Yes & 4 & 5.8 \\
No & & \\
\hline
\end{tabular}

Source: Field Survey, 2019

All the respondents $70(100 \%)$ stated yes health education provided by health worker has influenced them to adhere to childhood immunisation schedule. Respondents were asked if they had been able to complete or still running their children's immunization schedule childhood killer diseases and from Table 4, 66 (94.2\%) respondents said yes they had completed or still running children's immunization schedule and 4 (5.8) respondents said to they could not achieve it. 


\section{Discussion}

The age range selected for the study was within the female reproductive age group of 18-45. Marital status did not influence immunization schedule completion rate. Religion did not have any significance influence on completing child's immunisation schedule in this current study. There was an equal likelihood of being immunized completely despite the religious affiliations that participants belonged to in the study. Level of education is an essential predictor of outcome factors influencing immunization schedule completion. It is believed that mothers/ caregivers with higher knowledge levels are more likely to encourage immunization and do influence the attendance for immunisation. High level of trust in distributors of information and vaccines may be an essential explanatory factor as to why mothers or care givers seek immunisation for their children, although there may be costs. Respondents were asked if they have heard or seen messages about vaccination in the last month and all the $70(100 \%)$ respondents stated they always encounter them. It is suggested that heath education is also associated with increased self-efficacy and likely to offer exposure to preventive information.

Therefore, from the study, it revealed majority of the respondents $57(81.4 \%)$ respondents had a thorough knowledge on the need to immunise children against diseases. This calls for the need to offer health education on Child's immunisation through every available medium. This finding is consistent with that of Oyerinde (1999) who suggests in his study at Oyo State, Nigeria that there was a high level of knowledge about immunisation, the diseases prevented and the side effects. Similarly, World Bank (1993) notes that there is a positive correlation between the mother's education and child health in several studies was a driving factor underpinning the World Bank's campaign in the 1990s to encourage increased maternal education in developing countries.

All the respondents $(100 \%)$ indicated that immunisation is very important as it prevent childhood killer diseases. Nursing mothers possessing sufficient knowledge and understanding on immunisation intervention is very key in terms of attaining complete immunisation schedule of children particularly in developing countries. These results are consistent with that Bonsu (2005) who maintained that knowledge of the schedules of the antigens was high among the literates and that there is an increased percentage of a child being immunized if parents are well educated about the immunisation. All the respondents $(100 \%)$ mentioned that there was social mobilisation for immunisation schedule in their locality. Client's satisfaction is deemed to be an important explanatory factor as it seems to produce positive observed benefits. These results are in agreement with WHO's (2001) findings which showed that there is the need for training health workers to improve the local management of EPI services at all levels, strengthen the planning and implementation of IEC and social mobilisation activities, and consequently improve the demand for EPI services and community participation.

On the subject of the main source of information on immunisation schedule of respondents' children, 48 $(68.6 \%)$ obviously said health workers, $10(14.3 \%)$ said radio/FM, $8(11.4 \%)$ respondents said family members, $2(2.29 \%)$ respondents each said town announcers and friends respectively. This result further established what Srinivasan (1993) concluded that commonly, parents perceive health workers as a credible source of information about health). All the 70 (100\%) respondents held that they understood the Twi (Akan) used to educate them. In terms of the place respondents went for immunisation sessions, $63(90 \%)$ of the respondents mentioned Reproductive Child Health facility and 7 (10\%) respondents mentioned CHPS. Immunisation programmes and activities use many communication channels to reach parents and other target audiences, from radio and television, to folk media, community events to counselling at health facilities.

Moreover, in terms of the reason for incomplete vaccination among people in the respondents' community, these results seem to be consistent with other research which found some of the challenges encountered by the immunisation schedule in Ghana embrace geographical access to immunisation services, of awareness of caregivers on vaccination schedule, inadequate understanding and insufficient demand for immunization by families and communities, inadequate health staff to provide services to very large and scattered communities, inconsistent and inadequate performance by health workers and insufficient allocation and delayed financial support for immunisation services (Ministry of Health, 2002).

It is well established that information, education and communication about immunisation targeting mothers, tracking of defaulters significantly increases mothers' adherence to immunization program. All the respondents $70(100 \%)$ stated that health education provided by health worker has influenced them to adhere to childhood immunisation schedule and $66(94.2 \%)$ respondents stated that they had completed or still running children's immunisation schedule and $4(5.8 \%)$ respondents said to they could not achieve it. These results agree with the findings of other studies, in which argued that health interventions when implemented with community participation and tuned to meet the cultural and social context of the community are more successful (Scott, 2001). Informed patients, working together with their healthcare practitioners, are the main players in keeping themselves and their family healthy and protecting the health of the public.

Overall these, I argue that health workers should be critical in assisting to improve confidence in vaccines and to support public feel good about their choice to be vaccinated or to have their children or relatives to be vaccinated. There is the need on information about how immunisations functions in the human body, how vaccine safety is constantly monitored, and how to successfully communicate about the risk of disease against 
the risk of a vaccine side effect.

\section{Conclusion}

This study has documented the effect of health education on mother adherence to childhood immunisation schedule in selected towns in the Upper Denkyira East Municipality. The main results of the study suggest that health education has positive impact on mother adherence to childhood immunisation schedule. Reproductive and Child Health/Family Planning staff should embark on routine education campaigns at both antenatal and postnatal sessions and child welfare clinics stressing the total number of times mothers need to visit the clinic to complete the immunization. Our findings suggest that intensified outreach and regular home visits should be employed by community health nurses specifically for mothers who default as a result of obstacles and Ghana Health Service should ensure that incentive packages, thus allowances and accommodation are provided to motivate staffs who accept duties in hard-to-reach areas are disbursed in time.

The However, the current study has some limitations. First, the study does not involve health workers to explain the role of health education on mother adherence to childhood immunisation schedule. Secondly, the study was done only in the Dunkwa-On-Offin, the capital of the Municipality and it could have involve other towns in the entire municipality. However, the findings were not compromised in any way.

$\begin{array}{ll}\text { Abbreviations/Acronyms } \\ \text { EPI } & \text { Expanded Program on Immunization } \\ \text { GDHS } & \text { Ghana Demographic and Health Survey } \\ \text { GHS } & \text { Ghana Health Service } \\ \text { MOH } & \text { Ministry of Health } \\ \text { RCH } & \text { Reproductive and Child Health } \\ \text { UNICEF } & \text { United Nations Children's Fund } \\ \text { WHO } & \text { World Health Organization }\end{array}$

\section{References}

Bonsu, G., Addoquaye, J. \& Owusu-Agyei, S. (2005). A Comprehensive Assessment of the Quality of Immunization in Techiman District

Ghana Health Service: EPI 5YPOW (2000-2006): A Profile Ghana's Expanded Programme on Immunization in Ghana. 2002. http://www.who.int/countries/gha/publications/EPI_Profile.pdf.Accessed 26 Apr 2017.

Ghana Health Service. Annual Report for the year (2012). Accra: Ghana Health Services; 2013.

Ghana Demographic and Health Survey. Key indicators, Accra. 2014.

Ghana Statistical Service (GSS), Ghana Health Service. Ghana Demographic and Health Survey 2008.

Ghana Health Service (2002). Ghana Expanded Programme on Immunization: Reducing Missed Opportunities.

Ghana Health Service (2003). The National EPI Integrated 5-Year Communication Plan 2003-2007

Machingaidze S, Wiysonge CS, Hussey GD (2013) Strengthening the Expanded Programme on Immunization in Africa: Looking beyond 2015. PLoS Med 10(3): e1001405. https://doi.org/10.1371/journal.pmed.1001405

Ministry of Health (2002). The Second Health Sector 5YPOW 2002-2006 Partnership for Health: Bridging the Inequalities Gap

Oyerinde K. (1999). National Programme on Immunization Patronage in Selected Local Government Areas of Oyo State.

Srinivasan, L. (1993). A Manual for Training Trainers in Participatory Techniques State of the World's Children 2006

WHO and UNICEF. Progress towards global immunization goals - 2011. Available: http://www.who.int/immunization_monitoring/data/SlidesGlobalImmunization.pdf. Accessed 8 February 2013.

Wilson, T. (2000). Factors influencing the immunization status of children in a rural setting Journal of Pediatric Health Care, 14, 117-121.

World Bank. World Development Report 1993: Investing in Health. New York: Oxford University Press, 1993

World Health Organization (1974). Handbook of Resolutions. World Health Assembly, Fourteenth plenary meeting: 23 May 1974. Geneva: World Health Organization; 1974.

World Health Organisation (2003). Ensuring the Quality of Vaccines at Country Level. Guidelines for Health Staff. Geneva: World Health Organization. WHO/V\&B/02.16.

World Health Organisation (2003). Increasing Immunization Coverage at the Health Facility Level. Geneva: World Health Organization. WHO/V\&B/02.27.

World Health Organization; WHO/EPI/Gen/ 93.11 Field Guide for the Ghana Immunisation Programme, GHS 2003. 
World Health Organization (2006). The World Health Report 2006: Working Together for Health. Geneva, Switzerland: WHO.

World Health Organisation (2019). Children: reducing mortality. Accessed on 19 September 2019 https://www.who.int/en/news-room/fact-sheets/detail/children-reducing-mortality

Wyss, K., Moto, D.M., Callewaert, B. (2003). Constraints to scaling-up health related interventions: the case of Chad, Central Africa. J IntDev; 15:87-100.

Wiysonge, C. S. (2015). Why Africa is lagging behind in child vaccination. The Conversation October 14, 2015. https://theconversation.com/why-africa-is-lagging-behind-in-child-vaccination-48699 